\title{
The Structure of the Bovine Protein Tyrosine Phosphatase Dimer Reveals a Potential Self-Regulation Mechanism ${ }^{\dagger,+}$
}

\author{
Lydia Tabernero, ${ }^{\S}$ Bornadata N. Evans," Patrick A. Tishmack," Robert L. Van Etten," and Cynthia V. Stauffacher*, \\ Departments of Biological Sciences and Chemistry, Purdue University, West Lafayette, Indiana 47907
}

Received February 16, 1999; Revised Manuscript Received June 15, 1999

\begin{abstract}
The bovine protein tyrosine phosphatase (BPTP) is a member of the class of low-molecular weight protein tyrosine phosphatases (PTPases) found to be ubiquitous in mammalian cells. The catalytic site of BPTP contains a $\mathrm{CX}_{5} \mathrm{R}(\mathrm{S} / \mathrm{T})$ phosphate-binding motif or P-loop (residues 12-19) which is the signature sequence for all PTPases. Ser19, the final residue of the P-loop motif, interacts with the catalytic Cys 12 and participates in stabilizing the conformation of the active site through interactions with Asn15, also in the P-loop. Mutations at Ser19 result in an enzyme with altered kinetic properties with changes in the $\mathrm{p} K_{\mathrm{a}}$ of the neighboring His72. The X-ray structure of the S19A mutant enzyme shows that the general conformation of the P-loop is preserved. However, changes in the loop containing His72 result in a displacement of the His72 side chain that may explain the shift in the $\mathrm{p} K_{\mathrm{a}}$. In addition, it was found that in the crystal, the protein forms a dimer in which Tyr131 and Tyr132 from one monomer insert into the active site of the other monomer, suggesting a dual-tyrosine motif on target sites for this enzyme. Since the activity of this PTPase is reportedly regulated by phosphorylation at Tyr131 and Tyr132, the structure of this dimer may provide a model of a self-regulation mechanism for the low-molecular weight PTPases.
\end{abstract}

Protein tyrosine phosphorylation is an essential component of the intracellular signal transduction pathways that regulate cell growth, proliferation, and differentiation $(1,2)$. The level of protein tyrosine phosphorylation in the cell reflects the coordination of the enzymatic activities of kinases and phosphatases that phosphorylate and dephosphorylate target proteins $(1,3)$. The protein tyrosine phosphatase (PTPase) $)^{1}$ family includes a large number of proteins that can be categorized as high-molecular weight PTPases, low-molecular weight PTPases, and dual-specificity PTPases $(4,5)$. The low-molecular weight PTPases $\left(M_{\mathrm{r}} \sim 18000\right)$ are cytoplasmic and found to be ubiquitous in mammalian cells $(6-10)$. The sequences of this class of small enzymes exhibit no apparent homology with the high-molecular weight family except for the PTPase signature sequence, $\mathrm{CX}_{5} \mathrm{R}(\mathrm{S} / \mathrm{T})$, a structural motif that forms a phosphate binding site equivalent to the P-loop (phosphate-binding loop) in the high-molecular weight phosphatases.

This research was supported by National Institutes of Health Grant GM 27003 and by American Cancer Society Grant NP-946. X-ray facilities were supported in part by the Purdue Cancer Center.

$¥$ Coordinates have been deposited in the Protein Data Bank (file name 1COE).

* To whom correspondence should be addressed: Department of Biological Sciences, Lilly Hall of Life Science, Purdue University, West Lafayette, IN 47907-1392. Phone: (765) 494-4937. Fax: (765) 4961189. E-mail: cyndy@gauguin.bio.purdue.edu.

$\S$ Department of Biological Sciences.

"Department of Chemistry.

${ }^{1}$ Abbreviations: PTPase, phosphotyrosine phosphatase; BPTP, bovine low-molecular weight phosphotyrosine phosphatase; HCPTPA and HCPTPB, two isoenzymes of human low-molecular weight phosphotyrosine phosphatase; MES, 4-morpholineethanesulfonic acid; HEPES, $N$-(2-hydroxyethyl)piperazine- $N$-2-ethanesulfonic acid.
The structure of the bovine protein tyrosine phosphatase (BPTP) $(11-13)$ reveals a fold characterized by a highly twisted central $\beta$-sheet with $\alpha$-helices packed on both sides. The overall structure has the characteristics of a classic dinucleotide binding or Rossmann fold, with two clear righthanded $\beta \alpha \beta$ motifs contributing to the central four-strand parallel $\beta$-sheet. The active site in BPTP is formed by a loop containing the signature sequence that connects the $\mathrm{C}$ terminus of the first $\beta$-strand to the $\mathrm{N}$-terminus of the first $\alpha$-helix. A phosphate ion sits at the center of the loop nestled between Cys12 and Arg18 and hydrogen-bonded to the amide groups of residues in the loop. Although this loop has a sequence different from that normally observed in other phosphate binding motifs (12-CLGNICRS-19 compared to the canonical GXGXXG), it adopts a similar geometry with all the backbone amide groups oriented toward the phosphate ion. This is possible because Asn15 (conserved in the lowmolecular weight PTPases) adopts a left-handed helical conformation. Interestingly, Asn 15 makes hydrogen bonds with three other conserved residues, Ser19, Ser43, and His72, that apparently help to stabilize its left-handed conformation. Thus, it appears that Asn15, Ser19, His72, and Ser43 serve structural functions that allow the active site to adopt an optimal geometry for substrate binding and transition state stabilization.

Site-directed mutagenesis has been used to explore the role of some of these conserved residues involved in forming the P-loop of BPTP (14). The mutation of Ser19 to alanine resulted in an enzyme with altered kinetic properties, including a marked dependence on the leaving group $\mathrm{p} K_{\mathrm{a}}$ of the substrates that were tested. These experiments suggested that the formation of the phosphoenzyme intermediate becomes at least a partially rate-limiting step in this mutant, 
in contrast to the kinetic behavior of the wild-type enzyme for which dephosphorylation of the phosphoenzyme is entirely rate-limiting. It was therefore proposed that Ser19 acts to facilitate the ionization and orientation of Cys 12 for optimal reaction as a nucleophile. We report here the structure of the S19A mutant of BPTP at $2.2 \AA$ resolution and discuss the changes observed and their implications in the altered kinetic behavior of this mutant. In the crystal, we also observe that the two monomers in the asymmetric unit form a dimer with the active sites facing each other. Hydrogen bond interactions occur across the dimer interface, and we will analyze their significance for regulation and substrate specificity.

\section{EXPERIMENTAL PROCEDURES}

Protein Purification. Purification of S19A was achieved in two steps, involving ion exchange chromatography and gel filtration chromatography (14). The cell lysate was loaded onto a SP-Sephadex C-50 cation exchange column equilibrated with low-ionic strength buffer [10 $\mathrm{mM}$ sodium acetate, $10 \mathrm{mM} \mathrm{NaH}_{2} \mathrm{PO}_{4}$, and $1 \mathrm{mM}$ EDTA (pH 5.0)]. The mutant enzyme was eluted with $300 \mathrm{mM} \mathrm{NaH} \mathrm{PO}_{4}, 1 \mathrm{mM}$ EDTA, pH 5.1 buffer. The fractions containing the highest $A_{280}$ and specific activity were pooled, concentrated, and further purified using a Sephadex G-50 size exclusion column equilibrated with the same low-ionic strength buffer as the C-50 column.

Crystallization Conditions and Structure Determination. The protein sample was kept in the low-ionic strength buffer and further concentrated to $5 \mathrm{mg} / \mathrm{mL}$. Initial crystals for S19A were grown in $30 \% \mathrm{PEG}_{4000}$ and $0.1 \mathrm{M}$ Tris at $\mathrm{pH} 7.0$ in 2 days. Two steps of macroseeding were necessary to improve the size and quality of the crystals. Data were collected using an R-Axis II detector at room temperature on a plate-like crystal $(0.1 \mathrm{~mm} \times 0.2 \mathrm{~mm} \times 0.05 \mathrm{~mm})$ that diffracted up to $2.0 \AA$. The data from the S19A mutant were processed with Denzo (15) and indexed as a primitive orthorhombic cell $\left(P 2_{1} 2_{1} 2_{1}\right)$ with the following dimensions: $a=43.32 \AA, b=55.47 \AA, c=131.45 \AA$, and $\alpha=\beta=\gamma$ $=90.0^{\circ}$, with two molecules in the asymmetric unit $\left(V_{\mathrm{M}}=\right.$ $2.12 \AA^{3} / \mathrm{Da}$ ). In contrast, the native BPTP crystals belong to space group $C 2$ with the following unit cell dimensions: $a$ $=95.3 \AA, b=43.3 \AA, c=41.2 \AA$, and $\beta=113.5^{\circ}$. After being scaled with Scalepack (15), the data from 30 to $2.2 \AA$ have an $R_{\text {sym }}$ of $6.1 \%$ for 15625 unique reflections at the zero $\sigma$ level, and are $93.4 \%$ complete. The structure was solved by the molecular replacement method using AMoRe (16) with the native BPTP coordinates as a starting model (11). The self-rotation function exhibited a peak at $\psi=45^{\circ}$, $\phi=0^{\circ}$, and $\kappa=180^{\circ}$, corresponding to the pseudo-2-fold axis that relates the two molecules in the asymmetric unit. The resulting solution in the rotation and translation functions for the two molecules in the asymmetric unit exhibits a compact packing in the cell and an $R$ value of $48 \%$. Refinement was carried out using the program X-PLOR with the parameters of Engh and Huber $(17,18)$. Rigid-body refinement followed by energy minimization dropped the $R$ value from 48 to $35 \%$. Non-crystallographic symmetry constraints were applied in the initial steps of the refinement for the resolution range of 10-3.0 $\AA$. Simulated annealing and positional refinements were used in combination with manual fitting of the initial model. In a second stage, the resolution was increased up to $2.2 \AA$ and non-crystallographic restraints were then applied. Refinement was finished using CNS with the maximum likelihood target (19) to a final $R_{\text {work }}$ of $19.1 \%$ and an $R_{\text {free }}$ of $24.4 \%$ for data in the range of $20-$ $2.2 \AA$ with 13757 reflections. The final model contains 1529 atoms (residues 4-157) and 113 water molecules. The rmsd for bond distances is $0.006 \AA$ and for bond angles is $1.3^{\circ}$. The rmsd between the two molecules (all atoms except hydrogens) in the asymmetric unit is $0.18 \AA$.

After the initial rigid-body refinement, the $2\left(F_{\mathrm{o}}-F_{\mathrm{c}}\right)$ maps at the $2.5 \sigma$ level indicated significant shifts in the positions of the side chains of residues His72 and Trp49, both in the active site region. The indole ring of Trp49 in the S19A structure is displaced about $90^{\circ}$ with respect to the wildtype structure, due to the packing of the second monomer against the active site. The new position of Trp49 was modeled manually into the electron density maps. One run of simulated annealing refinement resulted in better quality maps and a rearrangement of the extended loop that contains His72 (residues 66-79).

${ }^{1} H$ NMR Spectroscopy. A sample of S19A was prepared for NMR spectroscopy by exchanging the G-50 column buffer for $99.9 \% \mathrm{D}_{2} \mathrm{O}, 20 \mathrm{mM} \mathrm{NaH} \mathrm{PO}_{4}$, and $130 \mathrm{mM} \mathrm{NaCl}$. The final volume was $0.6 \mathrm{~mL}$, and the concentration of the protein was $1.7 \mathrm{mM}$. The ${ }^{1} \mathrm{H}$ NMR spectra were obtained on a Varian VXR-600S spectrometer at $25{ }^{\circ} \mathrm{C}$ using the MLEV-17 pulse sequence for filtering out amide proton resonances (20). Each spectrum was acquired with a 7200 $\mathrm{Hz}$ spectral window, a $1 \mathrm{~s}$ acquisition time, a $1 \mathrm{~s}$ delay time, 128 acquisitions, a $10 \mathrm{~ms}$ MLEV-17 spin-lock time, and a $19 \mathrm{~Hz}$ field strength transmitter saturation of the residual HOD resonance, and processed with a 0.2 s Gaussian apodization function. The titration was performed in 13 steps from $\mathrm{pH} 4.8$ to 10 using small aliquots of a $100 \mathrm{mM} \mathrm{NaOD}$ or $100 \mathrm{mM} \mathrm{DCl}$ solution. The chemical shifts of the histidine $\mathrm{C}_{2} \mathrm{H}$ and/or the $\mathrm{C}_{5} \mathrm{H}$ resonances of His66 and His72 were monitored to determine the imidazole $\mathrm{p} K_{\mathrm{a}} \mathrm{s}$. The $\mathrm{p} K_{\mathrm{a}}$ values were determined from the modified Hill equation using a nonlinear least-squares fitting algorithm (21).

\section{RESULTS AND DISCUSSION}

Overall, the structure of the S19A mutant of bovine protein tyrosine phosphatase exhibited no large conformational changes when compared with the wild-type structure (11). For the main chain atoms, the rms deviation between both structures is $0.47 \AA$. The larger deviations were localized in exposed loop regions (Figure 1), in particular in residues $66-79$ between the second and third helix. The conformation of the P-loop remained the same as the wild-type enzyme with a rms deviation for the main chain atoms in the P-loop region (residues 12-19) of $0.32 \AA$. However, subtle differences in the positions of residues around the active site provided information that is important for understanding the kinetic and biophysical properties of the S19A mutant.

Shift in the His72 Side Chain Position and the Effect on the $p K_{a}$ of His72. In the wild-type BPTP enzyme, both His66 and His72 have unusually high $\mathrm{p} K_{\mathrm{a}}$ values, 8.36 and 9.19, respectively (22). On the basis of the initial X-ray BPTP structure (11), it was proposed that two acidic residues (Glu23 and Asp42) located near His72, and an acidic residue (Glu139) near His66, are primarily responsible for altering 


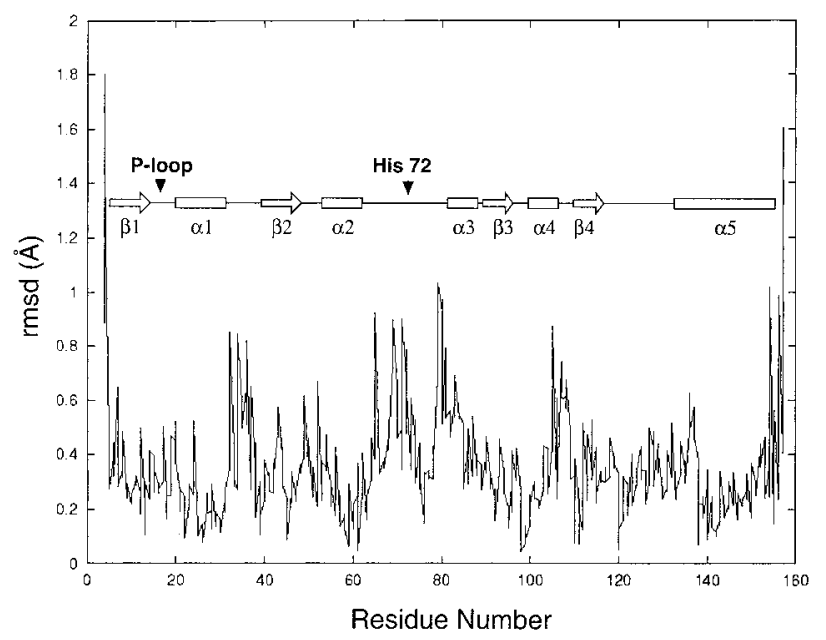

FIGURE 1: Plot of the backbone rms deviations between the BPTP wild-type and the BPTP S19A mutant structures. Superimposed is a graph of the topology of the polypeptide chain with the P-loop and the position of His 72 indicated. The larger differences between the backbone of both structures are located in the loop connecting the second and third helices (residues 66-79). This loop contains the His72 involved in hydrogen bonding interactions with residues in the P-loop. In contrast, the P-loop region that contains the S19A mutation exhibits a low rmsd between the wild-type and the mutant structure $(0.2-0.5 \AA)$.
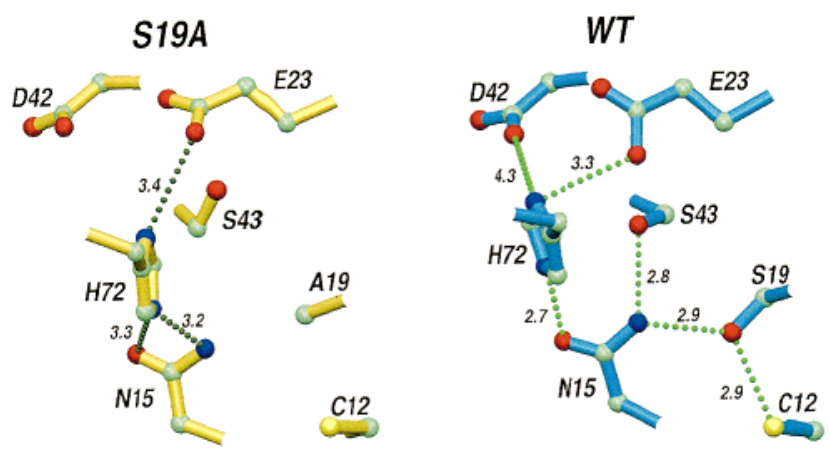

FIGURE 2: Comparison of the active sites of S19A (yellow) and wild-type (blue) BPTP structures. The interactions between His72 and neighboring residues, Asn15, Asp42, and Glu23, are drawn with all distances of $<4.5 \AA$ shown for comparison purposes. The hydrogen bond network around Asn15 in the wild-type enzyme is shown on the right. Note that this network is disrupted in the mutant, in which Asn 15 retains contact only with His72. This figure was prepared with SETOR (34).

the $\mathrm{p} K_{\mathrm{a}}$ values of these two residues. Histidines His66 and His 72 are highly conserved in the low-molecular weight PTPase family. Site-directed mutagenesis studies have shown that the His72 to Ala mutant loses about $75 \%$ of the wildtype activity (22), consistent with the location of this residue near the active site. An examination of the wild-type X-ray structure provides an explanation for the importance of His72, which is involved in a hydrogen bond network around Asn15, a residue in the P-loop region (11) (Figure 2). In the wild-type structure, interactions of Asn 15 with the $\mathrm{OH}$ group of Ser19, the $\mathrm{OH}$ group of Ser43, and the $\mathrm{N} \epsilon 2$ of His 72 were proposed to stabilize its left-handed backbone conformation. With Asn15 in this orientation, the P-loop remains a rigid open loop that is optimal for substrate binding and transition state stabilization. The structure of S19A reveals that the hydrogen bond network around Asn15 is disrupted, with a loss of interaction with Ser43 as well as the contact with Cys12 through the bridging hydrogen bonds of Ser19.
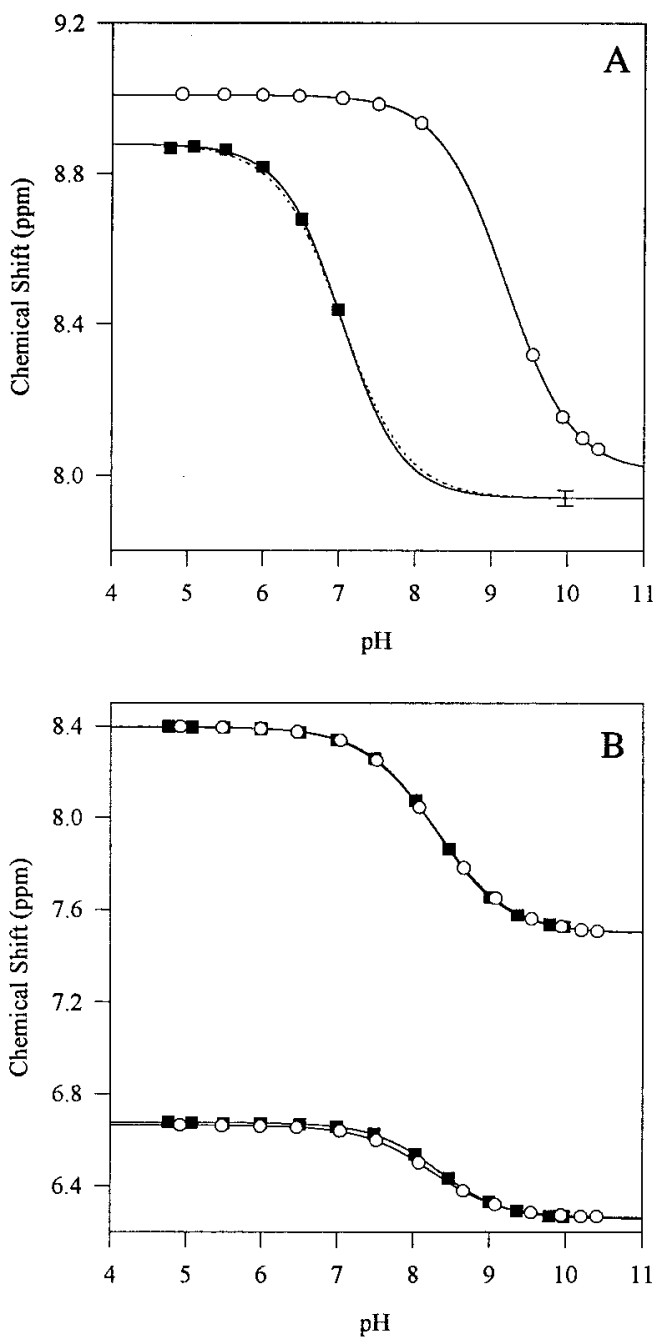

FIGURE 3: Comparison of the pH titration curves of His72 (A) and His66 (B) in wild-type BPTP (O) and S19A (ם). The chemical shift positions of the His $72 \mathrm{C}_{2} \mathrm{H}$ resonances were used to obtain the data points in panel A. For panel B, the upper two sets of data points were from His66 $\mathrm{C}_{2} \mathrm{H}$ resonances and the lower ones were from $\mathrm{C}_{5} \mathrm{H}$ resonances. The wild-type BPTP data are from a previously published work (21). The solid lines were calculated by nonlinear least-squares fitting of the observed data to the modified Hill equation. All the points on the S19A curve in panel A were experimentally observed values except that at $\mathrm{pH} 10$. This point was estimated on the basis of the mean chemical shift difference $(0.93 \pm 0.02 \mathrm{ppm})$ between the $\mathrm{C}_{2} \mathrm{H}$ resonance in the doubly protonated and singly protonated states of the His72 imidazole group for wild-type BPTP and 10 other mutants. The dotted line in panel $\mathrm{A}$ is the fitted curve obtained for a Hill coefficient of 1.0. The decrease in $\mathrm{p} K_{\mathrm{a}}$ as well as the shift to a lower frequency for His72 in S19A is readily observed in panel A.

Although His72 remains in contact with Asn15, it has also moved, but the overall P-loop conformation is not appreciably altered (Figure 2).

In the S19A mutant, His66 retains its high $\mathrm{p} K_{\mathrm{a}}$ (8.3). However, a striking change in the $\mathrm{p} K_{\mathrm{a}}$ of His 72 is observed. This $\mathrm{p} K_{\mathrm{a}}$ decreases from 9.2 in the wild-type enzyme to 6.9 in S19A, as estimated from $\mathrm{pH}$ titration curves (Figure 3). A detailed examination of the wild-type and S19A mutant enzyme structures reveals some interesting differences that may explain the shift in the $\mathrm{p} K_{\mathrm{a}}$ values of His72. In the $\mathrm{S} 19 \mathrm{~A}$ structure, there is a displacement of the long loop connecting the second and third helices (residues 66-79) (Figure 4). This displacement causes a shift in the position of the His 72 

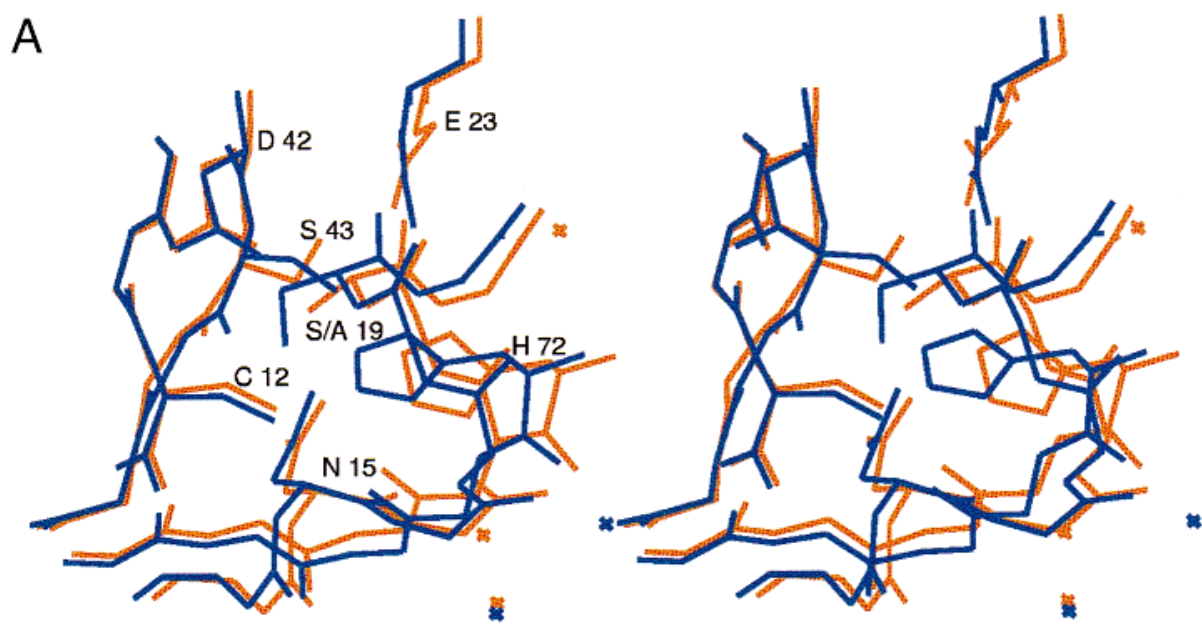

*

驾

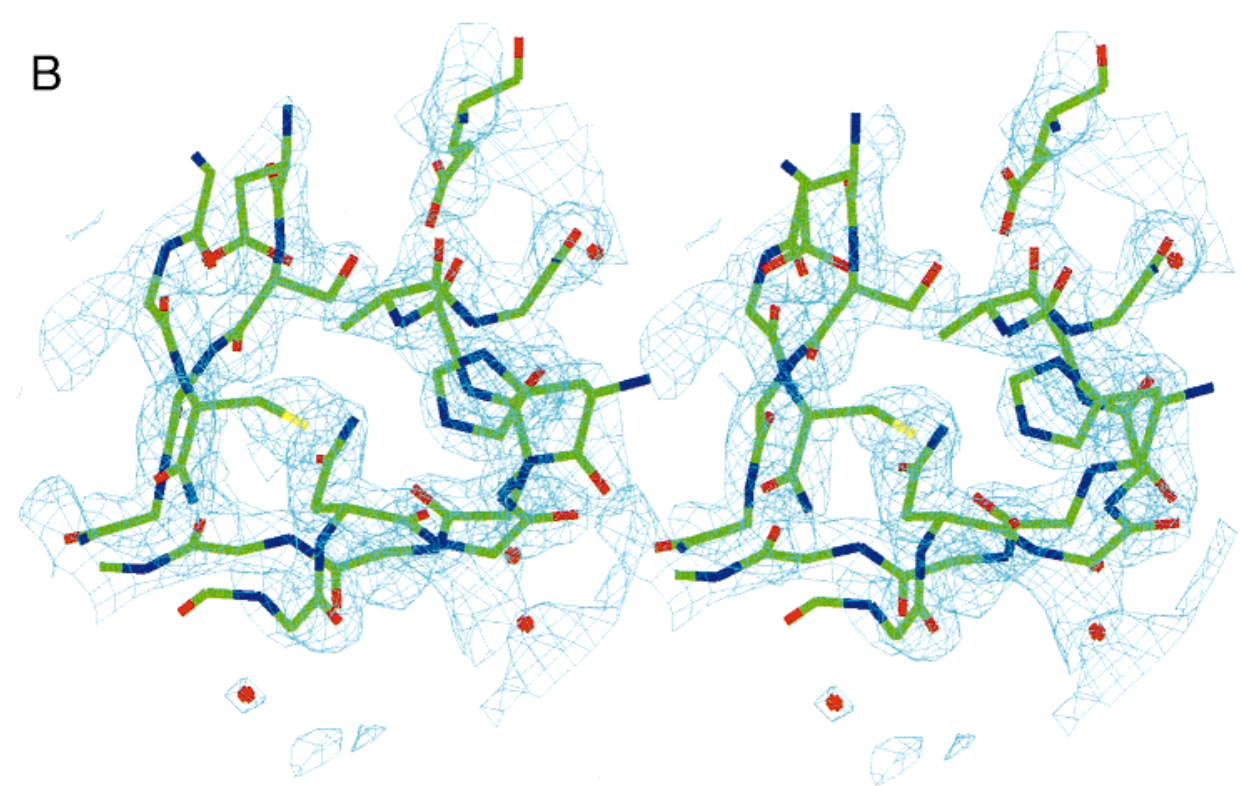

FIGURE 4: (A) Stereo representation of the superposition of the BPTP wild-type (blue) and S19A (orange) structures at the active site region. Note the displacement of the His72 side chain that results in a shift of this residue away from acidic residues Asp42 and Glu23. (B) Stereo representation of this same portion of the model in the active site region of S19A with the electron density map $\left(2 F_{\mathrm{o}}-F_{\mathrm{c}}\right)$ superimposed and contoured at the $2 \sigma$ level. Water molecules are shown as asterisks. Some P-loop residues have been removed for clarity. These comparisons show minor changes in the P-loop backbone, with Cys 12 retaining its position, roughly centered under the P-loop. The largest changes can be seen in the region surrounding His72. This figure was prepared with SETOR (34).

$\mathrm{C} \alpha$ of about $0.8 \AA$ and a shift in the position of the imidazole ring atoms of $1-2 \AA$. As a consequence of this displacement, the imidazole ring of His72 is further away from the two acidic residues, Asp42 and Glu23, proposed to be responsible for the increased $\mathrm{p} K_{\mathrm{a}}$ of His72 in the wild-type enzyme (Table 1). In addition, the $\mathrm{N} \epsilon 2$ atom of His 72 is now displaced toward the amino group of Asn15, occupying a midway position between the amino group and the carbonyl group. In the wild-type structure, the $\mathrm{N} \epsilon 2$ atom of His 72 was closer to the carbonyl oxygen of Asn15 (Figure 2). Overall, the imidazole ring of His72 in the S19A structure is considerably more distant from acidic residues Asp42 and Glu23 and hydrogen bond partners (like Asn15) than in the wild-type structure, which will minimize the electrostatic interactions with these neighbors and explain a drop in the $\mathrm{p} K_{\mathrm{a}}$. This is consistent with the fact that removal of the carboxylate groups from Asp42 and Glu23 in the double-
Table 1: Distance Comparisons between the His72 Imidazole Amide Groups and Neighboring Acidic Groups in the Active Site of Wide-Type and S19A Mutant BPTP Structures

\begin{tabular}{cccc}
\hline His72 & Glu23 & $\begin{array}{c}\text { wild-type } \\
\text { distance }(\AA)\end{array}$ & $\begin{array}{c}\text { S19A } \\
\text { distance }(\AA)\end{array}$ \\
\hline ND1 & OE1 & 3.88 & 5.08 \\
ND1 & OE2 & 3.29 & 3.37 \\
NE2 & OE2 & 4.25 & 4.73 \\
\hline \multirow{2}{*}{ His72 } & Asp42 & distance $(\AA)$ & distance $(\AA)$ \\
\hline ND1 & OD1 & 4.28 & 6.14 \\
NE2 & OD1 & 4.73 & 6.89 \\
\hline
\end{tabular}

mutant enzyme E23A/D42A results in a $\mathrm{p} K_{\mathrm{a}}$ for His72 of $6.2(21)$.

Role of the Ser19 OH Group. Steady state kinetic measurements revealed that the S19A mutant enzyme had 
substantially altered catalytic activities toward $p$-nitrophenyl phosphate and phenyl phosphate and exhibited $V_{\max }$ values that are 0.33 and $0.25 \%$ of that of the wild-type enzyme, respectively (14). Further kinetic analyses of the S19A mutant were performed using phosphomonoester substrates with varied phenolic leaving groups. The slope of the correlation between $V_{\max }$ and the substrate leaving group $\mathrm{p} K_{\mathrm{a}}$ was significantly altered, consistent with a change in the ratedetermining step from breakdown of the phosphoenzyme intermediate to formation of the phosphoenzyme. These and related experiments were interpreted to indicate that in the wild-type enzyme Ser19 acts to facilitate the ionization and orientation of Cys12 to permit optimal reaction of the thiolate as a nucleophile. It was proposed that upon disruption of the hydrogen bond interaction with Ser19, the ionized form of Cys 12 was no longer stabilized and might change its orientation away from an optimal position for nucleophilic attack on the substrate.

In the case of the Yersinia PTPase, a different role has been proposed for the conserved hydroxyl group in the signature motif (23). It was argued that Thr410 (which is in a position in the Yersinia PTPase structure similar to that of Ser19 in BPTP) is important in facilitating the breakdown of the phosphoenzyme intermediate. It was proposed that in the step that leads to the hydrolysis of the phosphoenzyme intermediate, the developing negative charge on the thiolate group in the transition state is stabilized by the hydroxyl group of Thr410. Such a role has also been proposed for the dual-specificity phosphatase VHR (24) and the Stp1 phosphatase (25).

The structure of the S19A mutant shows that although the hydrogen bond between Ser19 and Cys12 is disrupted because of the mutation, the Cys 12 side chain remains unaltered relative to the wild-type structure (Figure 2). Since no movement is observed, this suggests that Ser19 is primarily involved in the thiolate stabilization.

BPTP Dimer. The structure of the S19A mutant presents two molecules in the asymmetric unit related by a pseudo2 -fold axis (Figure 5A). The two molecules form a dimer in which the active sites are facing each other and residues Tyr131 and Tyr132 from one monomer insert into the active site cleft of the other monomer. The wild-type enzyme (BPTP) and the human low-molecular weight PTPase (HCPTPA) (26) were crystallized in the presence of HEPES and MES, aryl sulfonate inhibitory compounds that fill the active site with the sulfonate group bound in the P-loop. The S19A mutant enzyme was crystallized in the presence of phosphate; the phosphate ion binds in the P-loop, but the remaining cavity of the active site is unoccupied. We believe this is the reason the two tyrosines are able to bind into the active site, forming a stable dimer and changing the cell dimensions and the crystal habit from monoclinic in the wildtype crystals to orthorhombic in the mutant crystals.

To determine if dimer formation occurs and to assess the BPTP dimer stability in solution, we conducted preliminary sedimentation studies in a Beckman XLA analytical ultracentrifuge. Equilibrium ultracentrifugation experiments show that both the wild-type and S19A mutant enzymes exist at $\mathrm{pH} 4.8$ as a monomer $\left(M_{\mathrm{r}} \sim 17900\right)$ in the low-ionic strength buffer used in the protein purification (as described in Experimental Procedures). In contrast, at a more physiological pH of 7.3 in the crystallization Tris buffer, a monomer- dimer equilibrium was observed for both forms of the enzyme with an estimated equilibrium constant of approximately $1-5 \mathrm{mM}$ (results to be published). In this latter case, no phosphates which could stabilize the complex or aryl sulfonates which should compete with the dimerization are present. We believe that the dimer observed in solution in these experiments is the one which is captured in the S19A crystals grown at neutral $\mathrm{pH}$.

An analysis of the contacts in the dimer shows they involve residues that are important to specificity in the low-molecular weight PTPases. The two tyrosines (Tyr131 and 132) inserted in the active site establish specific interactions across the dimer interface. Tyr131 from one monomer forms a hydrogen bond through its hydroxyl group to Asn50 in the second monomer $(3.1 \AA$ for one active site and $3.4 \AA$ for the other), and Tyr 132 from one monomer forms a hydrogen bond through its hydroxyl group to Asp129, which is the general acid in catalysis, of the second monomer (2.5 and $2.6 \AA$ ) (Figure 5B). Additionally, the Trp49 side chain, which sits at the opening of the active site, rotates about $90^{\circ}$ from its original conformer in the wild-type structure to accommodate the second monomer (Figure 6).

Site-directed mutagenesis, steady state kinetics, and effector studies of the two human low-molecular weight PTPase isoenzymes (A form, HCPTPA, and B form, HCPTPB) indicated that residues 49 and 50 play important roles in determining the specificities of the two enzymes (26, 27). These two residues, Trp49 and Asn50, are located in a loop (the variable loop) at the outer rim of the active site. When Trp49 and Asn50 residues in the B form of the human enzyme (homologue to the BPTP enzyme) are mutated to their analogues in the A form, Tyr49 and Glu50, the $k_{\text {cat }} / K_{\mathrm{m}}$ value of the double mutant for phosphotyrosine is on the same order of magnitude as that of the A isoenzyme. Differing responses of the A and B forms of the enzyme toward activators and inhibitors have also been reported (28, 29). For example, residues 49 and 50 are involved in the strong activation of the B form by guanosine and cGMP. Mutations of Trp49 to its equivalent in the A form, a tyrosine, and Asn50 to a glutamate, result in enzymes with kinetic properties of the A form for cGMP activation (29).

The interactions of Tyr131 and Tyr132 in the dimer of this B form enzyme may be interpreted as mimicking specific interactions with natural substrates or inhibitors. In fact, the ring position of Tyr132, which interacts with the catalytic residue Asp129, is similar to the ring positions of the HEPES molecule in the BPTP structure (Figure 6) and the MES molecule in the HCPTPA structure in the active site (26). In the BPTP structure, the ring of HEPES packs parallel to the ring of Tyr131. In the HCPTPA structure, the ring of MES is perpendicular to Tyr131. In the dimer described here, Tyr132 from the first monomer packs perpendicular to the Tyr131 at the entrance of the active site of the second monomer and contacts Asp129, and Tyr131 from the first monomer points to the opposite wall of the active site and contacts Asn50 in the second monomer (Figure 6). If these interactions mimic a putative biological substrate, it is possible that the natural target also has multiple phosphotyrosines.

Dephosphorylation rates of a variety of phosphopeptide substrates derived from phosphorylation sites of cellular proteins have been measured using HCPTPA and 


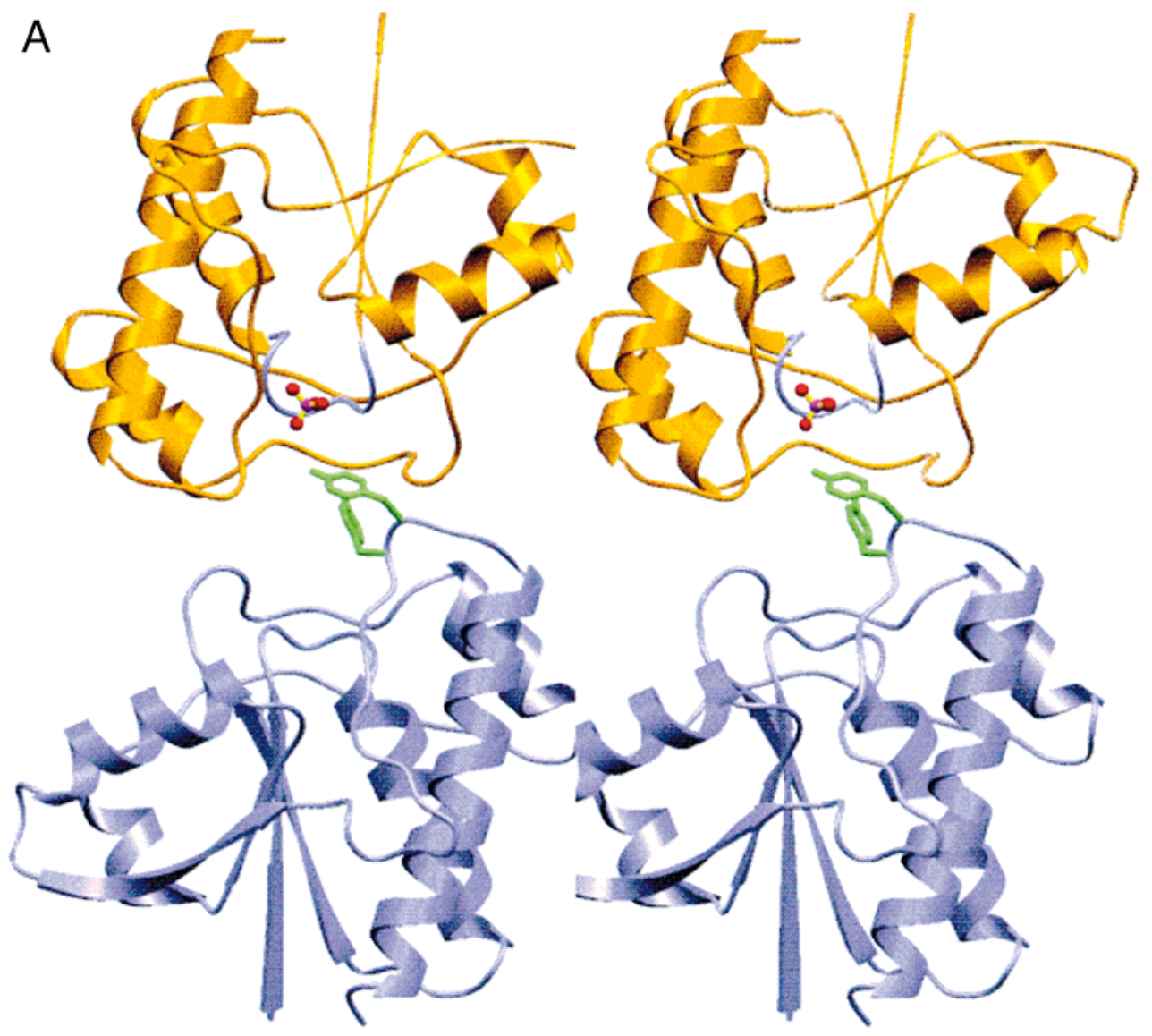

B

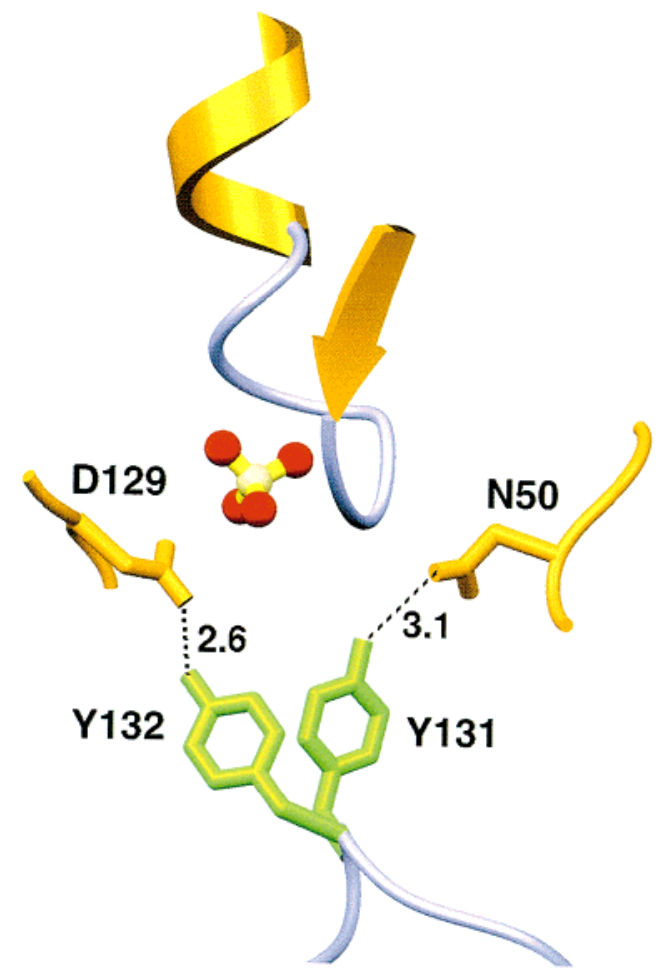

FIGURE 5: (A) Stereo ribbon representation of the two molecules in the asymmetric unit in the S19A crystal. The two monomers (yellow and purple) are related by a non-crystallographic 2-fold axis perpendicular to the plane of the paper. Tyr131 and Tyr132 are green. The P-loop in purple and bound phosphate are shown in the yellow monomer. It can be observed that the tyrosine residues from one monomer (purple) insert into the active site of the other monomer (yellow) in this non-crystallographic dimer. (B) Representation of the dimer interface near the active site P-loop. The hydrogen bond interactions of Tyr131 and Tyr132 (green) from one monomer (purple) and residues Asn50 and Asp129 of the other monomer (yellow) across the dimer interface are shown. This figure was prepared with SETOR (34).

HCPTPB (30). The results of these experiments show that both isoenzymes can dephosphorylate the SykYY peptide (DENY $\left.{ }_{\mathrm{p}}^{525} \mathrm{Y}_{\mathrm{p}}^{526} \mathrm{KAQ}\right)$ and that the SykYYY peptide $\left(\mathrm{LRNY}{ }_{\mathrm{p}}{ }^{629} \mathrm{Y}_{\mathrm{p}}{ }^{630} \mathrm{Y}_{\mathrm{p}}{ }^{631} \mathrm{DVN}\right)$ appears to be the best substrate 

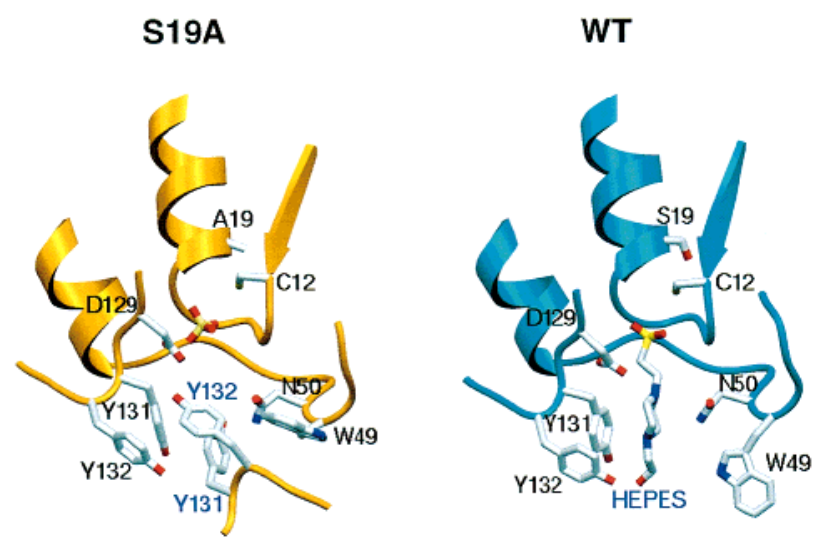

FIGURE 6: Comparison of the active site region of the BPTP wild type (blue) in complex with HEPES, seen with its ring packed against Tyr131, and the active site of S19A (yellow) with Tyr131 and Tyr132 inserted into the active site. The insertion of the loop containing Tyr131 and Tyr132 displaces the side chain of Trp49 on the side wall of the active site opening, but otherwise, the binding of the HEPES and the double tyrosine loop uses the same configuration of active site residues. This figure was prepared with SETOR (34).

among all the phosphopeptides that were tested. Furthermore, the $k_{\text {cat }} / K_{\mathrm{m}}$ value for the dephosphorylation of SykYYY is about 2 times greater for HCPTPB than for HCPTPA, which may indicate a further preference of this isoform for substrates with tandem phosphotyrosines. It has been reported that the rat low-molecular weight PTPase associates with the insulin receptor $\beta$-subunit and downregulates the mitogenic and metabolic insulin-mediated response in fibroblasts (31). Interestingly, the insulin receptor tyrosine kinase and the fibroblast growth factor receptor kinase both contain a tandem of two tyrosines in the activation loop which are phosphorylated in the active form of the kinase, and which may be a candidate target for this phosphatase. Double tyrosines are also found in the LTK/Alk family, hepatocyte growth factor, NKIN15/16, DDR/TKT, Ros/sevenless, Axl, Fak, Tyk, Jak, and Syk/Zap70 kinases.

Mechanism of Self-Regulation. Recent reports have suggested that this low-molecular weight phosphatase, as expressed in T-cells, is activated by phosphorylation at Tyr131 and to a lesser extent at Tyr132 (32, 33). Phosphorylation of the phosphatase may be accomplished by Lck or Fyn kinases in vivo. It was proposed that activation of T-cells upon formation of the antigen-receptor complex results in rapid dephosphorylation of this PTPase with subsequent inactivation of the enzyme. The insertion of Tyr131 and Tyr132 into the active site in the crystallized dimer suggests a way in which rapid dephosphorylation could occur with this enzyme serving as its own substrate. A model for selfregulation may be proposed as follows. In vivo, as a response to the appropriate signal, BPTP is phosphorylated and becomes activated. Phosphorylation at Tyr131 and at Tyr132 would cause a strong repulsion between the contiguous phosphate groups. This repulsion would force a conformational rearrangement of the loop that contains the tyrosines and result in a change at the entrance of the active site where they are located (open form). In this open conformation, the PTPase will be able to bind its natural phosphosubstrate and exert its function as a phosphatase. Subsequently, as the levels of phosphosubstrate decay, the PTPase will rapidly auto-dephosphorylate itself as a fast turn-off mechanism.
Upon dephosphorylation, the loop will change back to its unphosphorylated conformation, resulting in a narrower entrance of the active site (closed form) that would prevent binding to the target substrate. The PTPase will then enter into a resting inactive state. According to this model, the dimer in the crystal would represent a transient state between the desphophorylation of the tyrosines and the release of the unphosphorylated resting monomers. One could also argue that the dimer represents a product inhibition equilibrium state and will be displaced in the presence of the natural phosphosubstrate.

\section{ACKNOWLEDGMENT}

We acknowledge John W. Burgner II for providing the preliminary data on the ultracentrifugation studies. We also want to thank Marie Zhang, Guoguang Lu, and Germaine Sainz for their kind and helpful assistance with crystallization and refinement of the structure.

\section{REFERENCES}

1. Charbonneau, H., and Tonks, N. K. (1992) Annu. Rev. Cell Biol. 8, 463-493.

2. Fisher, E. H., Charbonneau, H., and Tonks, N. K. (1991) Science 253, 401-406.

3. Cohen, P. (1989) Annu. Rev. Biochem. 58, 453-508.

4. Zhang, M., Stauffacher, C. V., and Van Etten, R. L. (1995) Adv. Protein Phosphatases 9, 1-23.

5. Fauman, E. B., and Saper, M. A. (1996) Trends Biochem. Sci. $21,413-417$.

6. Heinrikson, J. L. (1969) J. Biol. Chem. 244, 299-307.

7. Rehkop, D. M., and Van Etten, R. L. (1975) Hoppe Seyler's Z. Physiol. Chem. 356, 1775-1782.

8. Chernoff, J., and Li, H. C. (1985) Arch. Biochem. Biophys. $240,135-145$.

9. Waheed, A., Laidler, P. M., Wo, Y.-Y. P., and Van Etten, R. L. (1988) Biochemistry 27, 4265-4273.

10. Zhang, Z.-Y., and Van Etten, R. L. (1990) Arch. Biochem. Biophys. 282, 39-49.

11. Zhang, M., Van Etten, R. L., and Stauffacher, C. V. (1994) Biochemistry 33, 11097-11105.

12. Su, X.-D., Taddel, N., Stefani, M., Ramponi, G., and Nordlund, P. (1994) Nature 370, 575-578.

13. Logan, T. M., Zhou, M.-M., Nettesheim, D. G., Meadows, R. P., Van Etten, R. L., and Fesik, S. W. (1994) Biochemistry 33, 11087-11096.

14. Evans, B., Tishmack, P., Pokalsky, C., Zhang, M., and Van Etten, R. L. (1996) Biochemistry 35, 13609-13617.

15. Otwinowski, Z. (1993) in Proceedings of the CCP4 Study Weekend: Data Collection and Processing (Sawyer, L., Isaacs, N., and Baily, S., Eds.) pp 56-62, SERC, Daresbury, U.K.

16. Navaza, J. (1987) Acta Crystallograph. A43, 645-653.

17. Brünger, A. T., Kuriyan, J., and Karplus, M. (1987) Science 235, 458-460.

18. Engh, R. A., and Huber, R. (1991) Acta Crystallogr. A47, $392-400$.

19. Brünger, A. T., Adams, P. D., Clore, G. M., DeLano, W. L., Gros, P., Grosse-Kunstleve, R. W., Jiang, J. S., Kuszewski, J., Nilges, M., Pannu, N. S., Read, R. J., Rice, L. M., Simonson, T., and Warren, G. L. (1998) Acta Crystallogr. D54, 905-921.

20. Zhou, M.-M., Davis, J. P., and Van Etten, R. L. (1993) Biochemistry 32, 8479-8486.

21. Tishmack, P. A., Bashford, D., Harms, E., and Van Etten, R. L. (1997) Biochemistry 36, 11984-11994.

22. Davis, J. P., Zhou, M.-M., and Van Etten, R. L. (1994) Biochemistry 33, 1278-1286.

23. Zhang, Z.-Y., Palfey, B. A., Wu, L., and Zhao, Y. (1995) Biochemistry 34, 16389-16396. 
24. Denu, J., and Dixon, J. (1995) Proc. Natl. Acad. Sci. U.S.A. 92, 5910-5914.

25. Zhao, Y., and Zhang, Z.-Y. (1996) Biochemistry 35, 1179711804.

26. Zhang, M., Stauffacher, C. V., Lin, D., and Van Etten, R. L. (1998) J. Biol. Chem. 273, 21714-21720.

27. Evans, B. (unpublished results).

28. Dissing, J., Rangaard, B., and Christensen, U. (1993) Biochim. Biophys. Acta 1162, 275-282.

29. Cirri, P., Fiaschi, T., Chiarugi, P., Camici, G., Manao, G., Raugei, G., and Ramponi, G. (1996) J. Biol. Chem. 271, 2604-2607.
30. Schroff, A. D. (1997) Ph.D. Thesis, Purdue University, West Lafayette, IN.

31. Chiarugi, P., Cirri, P., Marra, F., Raugei, G., Camici, G., Manao, G., and Ramponi, G. (1997) Biochem. Biophys. Res. Commun. 238, 676-682.

32. Tailor, P., Gilman, J., Williams, S., Couture, C., and Mustelin, T. (1997) J. Biol. Chem. 272, 5371-5374.

33. Rigacci, S., Degl'Innocenti, D., Bucciantini, M., Cirri, P., Berti, A., and Ramponi, G. (1996) J. Biol. Chem. 271, 1278-1281.

34. Evans, S. V. (1993) J. Mol. Graphics 11, 134-138.

BI990381X 